\begin{tabular}{|c|l|}
\hline Title & Inducible defenses in prey intensify predator cannibalism \\
\hline Author(s) & Kishida, Osamu; Trussell, Geoffrey C.; Nishimura, Kinya; Ohgushi, Takayuki \\
\hline Citation & $\begin{array}{l}\text { Ecology, 90(11), 3150-3158 } \\
\text { https://loi.org/10.1890/08 2158.1 }\end{array}$ \\
\hline Issue Date & $2009-11$ \\
\hline Doc URL & http://hdl.handle.net/2115/44963 \\
\hline Type & article \\
\hline File Information & 2009 Ecology.pdf \\
\hline
\end{tabular}

Instructions for use 


\title{
Inducible defenses in prey intensify predator cannibalism
}

\author{
Osamu Kishida, ${ }^{1}$ Geoffrey C. Trussell,${ }^{2,4}$ Kinya Nishimura, ${ }^{3}$ and Takayuki Ohgushi ${ }^{1}$ \\ ${ }^{1}$ Center for Ecological Research, Kyoto University, Otsu 520-2113 Japan \\ ${ }^{2}$ Marine Science Center, Northeastern University, 430 Nahant Road, Nahant, Massachusetts 01908 USA \\ ${ }^{3}$ Graduate School of Fisheries Sciences, Hokkaido University, Hakodate 041-8611, Hokkaido, Japan
}

Abstract. Trophic cascades are often a potent force in ecological communities, but abiotic and biotic heterogeneity can diffuse their influence. For example, inducible defenses in many species create variation in prey edibility, and size-structured interactions, such as cannibalism, can shift predator diets away from heterospecific prey. Although both factors diffuse cascade strength by adding heterogeneity to trophic interactions, the consequences of their interaction remain poorly understood. We show that inducible defenses in tadpole prey greatly intensify cannibalism in predatory larval salamanders. The likelihood of cannibalism was also strongly influenced by asymmetries in salamander size that appear to be most important in the presence of defended prey. Hence, variation in prey edibility and the size structure of the predator may synergistically affect predator-prey population dynamics by reducing prey mortality and increasing predator mortality via cannibalism. We also suggest that the indirect effects of prey defenses may shape the evolution of predator traits that determine diet breadth and how trophic dynamics unfold in natural systems.

Key words: behavior; cannibalism; diet; food webs; Hynobius retardatus (Dunn); inducible defense; predation risk; Rana pirica (Matsui); salamander; trait-mediated indirect effects; trophic cascade.

\section{INTRODUCTION}

Classical views of trophic dynamics used simple food chains to argue that carnivores strongly control herbivore biomass, which in turn, influences plant biomass (Hairston et al. 1960, Rosenzweig 1971, 1973, Oksanen et al. 1981, Carpenter et al. 1987). Such "green world" or "exploitation" hypotheses therefore emphasize the importance of top-down control, and some systems are clearly driven this way (Estes and Palmisano 1974, Silliman and Bertness 2002). However, it is also clear that such striking trophic cascades do not operate in many systems because the reticulate nature of food webs, as well as other factors, can buffer their influence (Strong 1992, Polis and Strong 1996). For example, intraspecific heterogeneity or differentiation (Strong 1992), such as variation in plant edibility or quality, can strongly determine how effectively one species harvests the biomass of another in the food web and, thus, the trophic dynamics of the system (also see Leibold 1989, Persson 1999, Vos et al. 2004b).

Heterogeneity within a species can arise in a number of ways, but two factors seem to be emerging in terms of their importance to population and community dynamics. First, there is often considerable variation in the edibility of species (Leibold 1989, Strong 1992), and much of this variation may arise because of inducible defenses. The ecological significance of inducible de-

Manuscript received 20 November 2008; revised 12 February 2009; accepted 3 March 2009. Corresponding Editor: E. D. Grosholz.

${ }^{4}$ Corresponding author. E-mail: g.trussell@neu.edu fenses is well established (Agrawal 2001), and theory suggests that such plasticity can promote species coexistence and stabilize population dynamics (Matsuda et al. 1993, Bolker et al. 2003) as well as provide insight into the paradox of enrichment (Vos et al. 2004a, b) and the complexity-stability debate (Kondoh 2007). Moreover, because inducible defenses occur in numerous plant and animal species (Tollrian and Harvell 1999), the heterogeneity they create is likely to produce effects that operate throughout natural food webs.

Second, many species are composed of different size cohorts (Polis 1984) and the size-structured interactions that emerge in these situations can also serve to increase trophic heterogeneity within the food web. Cannibalism is one form of size-structured interaction that is prevalent in many food webs (Polis 1981, Persson 1999), and it can lead to interactions and dynamics that are not considered or predicted by unstructured models (Persson et al. 2003, Rudolf 2007a, b, 2008). For example, in amphibian systems these different dynamics can emerge because indirect interactions between just two species are possible when size-structured interactions like cannibalism are operating (Rudolf 2006). Moreover, recent models (Rudolf 2007b) suggest that the indirect interactions resulting from cannibalism can modify trophic cascades as well as the effects of enrichment on population dynamics.

Although the ecological importance of inducible defenses and cannibalism is increasingly recognized, to our knowledge, no empirical study has examined how their combined effects may influence the outcome of species interactions in natural populations. In this paper, 
we show that induced morphological defenses in tadpole prey greatly intensify cannibalism among predatory salamanders. Moreover, asymmetries in the size of cannibalistic salamanders and conspecific prey also exerted a stronger influence on the probability of cannibalism in the presence of tadpole defenses. Hence, variation in the edibility of tadpole prey can exert strong trait-mediated indirect effects in this system, which may shape salamander trait evolution by increasing the likelihood of cannibalism.

\section{Materials And Methods}

\section{Study system and background}

Tadpoles of Japanese brown frogs (Rana pirica [Matsui]) and predatory larval salamanders (Hynobius retardatus [Dunn]) provide an excellent predator-prey system for studying the evolutionary and ecological significance of adaptive morphological plasticity (Kishida and Nishimura 2005, 2006). Both amphibian larvae exhibit antagonistic morphological plasticity in response to one another. Prey tadpoles typically develop bulgier bodies (Fig. 1a, left, Fig. 1b; see also Plate 1) by thickening their epithelium tissues in response to larval salamander risk cues (Kishida and Nishimura 2004). Because salamanders are gape-limited predators that swallow their prey whole, the bulgy morph is highly effective in reducing tadpole vulnerability to salamander predation because it is more difficult to swallow than non-induced tadpole morphs. In contrast, salamander larvae can produce a predaceous morph (Fig. 1a, right) having an enlarged gape that allows them to swallow larger prey (Michimae and Wakahara 2002). Although salamanders primarily consume tadpoles when they are available, they also can cannibalize conspecifics, and this diet shift is likely influenced by the prevalence of bulgy tadpoles, whose bodies are significantly wider than those of salamander conspecifics (Fig. 1b). Hence, we predicted that when salamanders cohabit with bulgy tadpoles, their difficulty in consuming this defended morph would indirectly intensify cannibalism among salamanders. We tested this and other hypotheses with field and laboratory experiments that compared tadpole and salamander survivorship and morphology when salamanders are present with either defended or undefended tadpoles.

\section{Field experiment in a natural pond}

Our field experiment was conducted in a mountain pond (area $=55 \mathrm{~m}^{2}$ ) located in Hekirichi, Hokuto, Hokkaido, Japan $\left(41^{\circ} 53^{\prime} \mathrm{N}, 140^{\circ} 34^{\prime} \mathrm{E}\right)$. This pond had a limited tree canopy, a maximum depth of $0.5 \mathrm{~m}$, and a soil bottom. Adult salamanders (Hynobius retardatus) and frogs (Rana pirica) typically begin spawning in April, and the larvae of both amphibians coexist and interact from spring to summer. Invertebrate predators such as dragonfly larvae were not very abundant; those that were present were restricted to patches of aquatic vegetation along the periphery of the pond. a
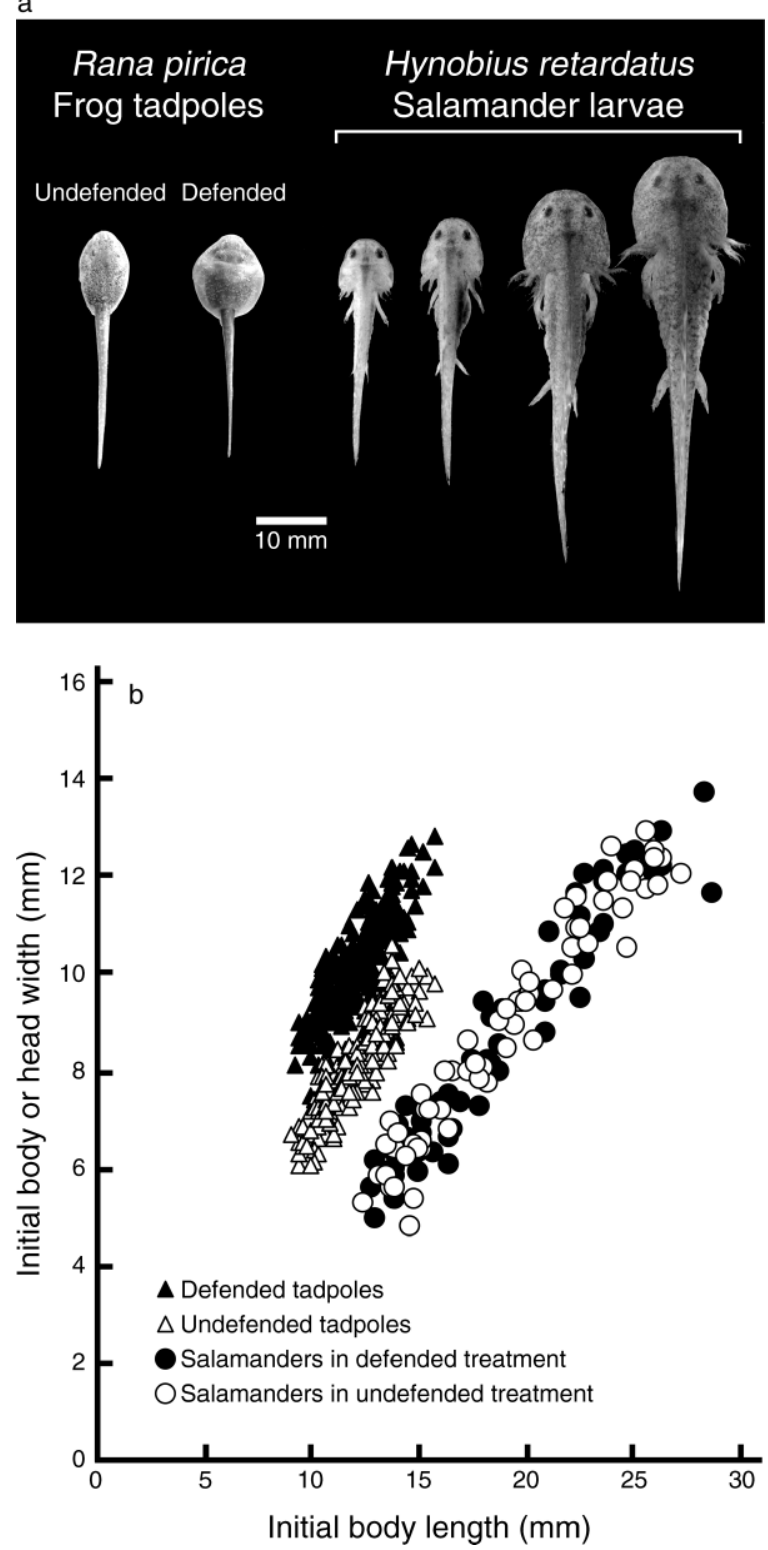

FIG. 1. Morphological variation in Rana pirica tadpoles and Hynobius retardatus salamanders. (a) Photos of (left) undefended and (right) defended frog tadpole phenotypes, and morphological variation of salamander larvae present in the experimental pond habitat ([left] non-predaceous small phenotype to [right] predaceous large phenotype). (b) Differences in the initial body width (defended and undefended tadpoles) and head width (salamanders) as a function of body length of amphibians used in our field experiment. Defended tadpoles having a bulgy body are induced by the presence of predatory larval salamanders and were collected from the pond where the two species coexist and our field experiment was conducted. Undefended tadpoles were collected from nearby ponds containing no larval salamanders. ANCOVA $\left(F_{3,720}=\right.$ 1823.12, $P<0.0001)$ and post hoc linear contrasts revealed that defended tadpoles are significantly wider than undefended tadpoles $(P<0.0001)$, and undefended tadpoles have bodies that are wider than salamander heads $(P<0.0001)$. 

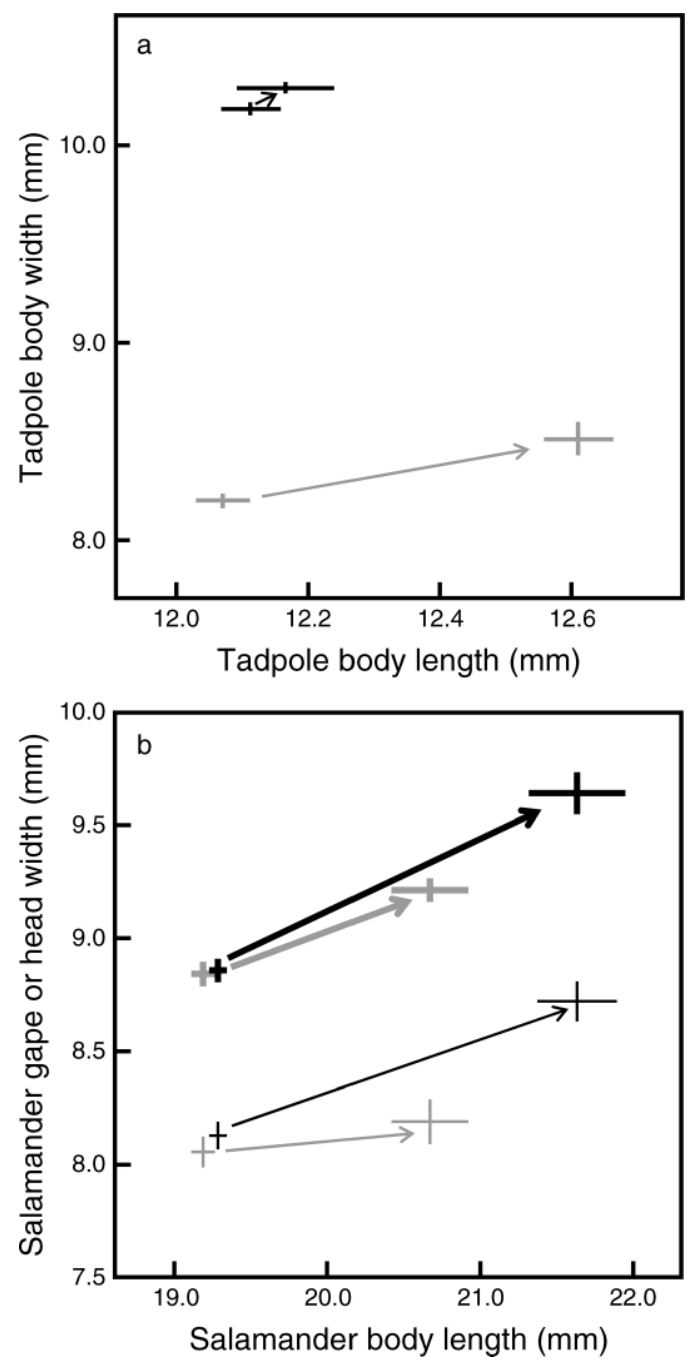

FIG. 2. (a) Body width (mean $\pm \mathrm{SE}$ ) as a function of body length for tadpoles and (b) gape width (mean \pm SE; thin lines) and head width (mean \pm SE; thick lines) as a function of length for salamanders at the beginning (base of arrows) and end of the field experiment (head of the arrows). Black crosses and lines represent the defended treatment, and gray crosses and lines represent the undefended treatment.

After spawning, frog and salamander eggs began to hatch from early to mid May. Soon after hatching, interactions between both amphibian larvae led to induced morphological changes as they grew. By early June, tadpoles had fully expressed their defensive bulgy morphs whereas the salamander population was composed of large individuals having a large gape (predaceous morph) and small individuals having a small gape (non-predaceous morph; Fig. 1a). Before initiating the field experiment, we first estimated the density of both amphibian larvae by randomly placing 10 quadrats $(80$ $\times 80 \mathrm{~cm}$ ) throughout the pond. Because it was difficult to reliably identify the species of each larva, this approach was only able to provide an estimate of overall amphibian larvae density $(89.8 \pm 21.1$, mean \pm
SD). Hence, we also used dip nets to collect many larvae and returned these samples to the laboratory to determine the proportion of tadpole and salamander larvae in the population and their size distributions. Defended tadpoles and salamanders collected from this pond were classified (in 1-mm categories) according to body length (snout-vent length), which is an appropriate measurement of size for the study of morphological plasticity in $R$. pirica and $H$. retardatus (Kishida and Nishimura 2005, Kishida et al. 2009). After size measurements, larvae were maintained individually in containers having $200 \mathrm{~mL}$ of aged tap water.

We also collected sufficient numbers of undefended tadpoles (basic morph) from several nearby ponds that contained no salamanders. These undefended tadpoles were conditioned to salamander predation risk for two days before initiating the experiment. We did so by randomly placing fifty undefended tadpoles into $13-\mathrm{L}$ tanks $(N=15)$ having $4 \mathrm{~L}$ of aged tap water and three, freely roaming salamander larvae to serve as inducers of predation risk. During this conditioning period, the undefended tadpoles became acclimated to predation risk as evidenced by their reduced activity levels, which is a common response to predation risk in many amphibian larvae species (Skelly 1994, Relyea 2001, Van Buskirk 2002, Kishida et al. 2009). Although tadpoles clearly responded behaviorally to salamander predation risk, they continued to maintain the noninduced morph because our conditioning period was too short to allow development of the bulgy morph. After this conditioning period, undefended tadpoles were also classified in 1-mm increments of body length (snoutvent length).

We placed eight replicate, rectangular $(80 \times 80 \times 80$ $\mathrm{cm}$ ) enclosures having PVC framing and nylon mesh (1mm openings) on all sides into the pond. We added $5 \mathrm{~L}$ of pond silt and sand on the mesh bottom of each enclosure to serve as natural substratum. Enclosures were stocked with natural density and size distributions of both amphibian larvae based on the sampling just described. We randomly applied two treatments ("undefended" and "defended") to the enclosures, and each treatment was replicated four times. In the undefended enclosures, we added 16 salamanders and 74 tadpoles having the undefended morph. In the defended enclosures, we added 16 salamanders and 74 tadpoles having the defended morph. All animals used in the experiment were measured for body length and width (tadpoles) or body length and gape and head width (salamanders) before placing them in the enclosures. Consistent with our experimental objectives, initial tadpole morphology (Fig. 2a) differed between defended and undefended treatments (MANOVA, $F_{1,6}=706.20, P<0.0001$ ) but a significant trait $\times$ treatment interaction (MANOVA, $\left.F_{1,6}=3764.81, P<0.0001\right)$ indicated that only body width $\left(F_{1,6}=2944.79, P<0.0001\right)$ and not body length $\left(F_{1,6}=1.27, P=0.3025\right)$ was different. There were no differences in the body length and gape and head width 


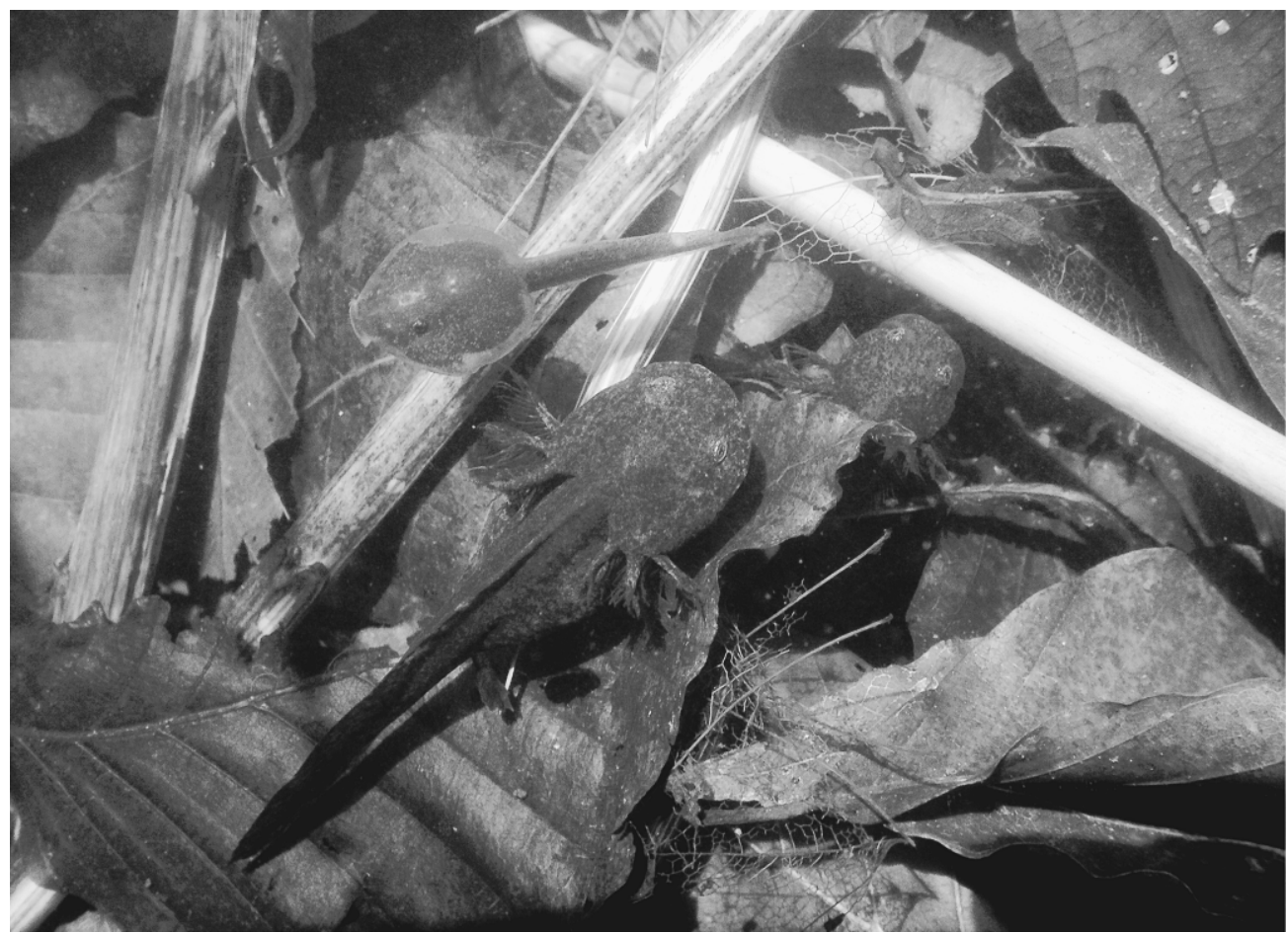

Plate 1. Predatory larval salamanders and frog tadpoles often co-occur in the mountain ponds of Japan. To reduce their risk of being eaten, tadpoles produce a defended ("bulgy") phenotype (upper left) that is difficult for salamanders (center) to swallow. From beneath the litter (upper right), a small non-predaceous salamander is also visible. The presence of defended tadpole morphs may ultimately lead to this small salamander being consumed by a large salamander. Photo credit: O. Kishida.

(MANOVA, $F_{1,6}=0.55, P=0.4845$; Fig. 2b) of salamanders placed in the defended and undefended enclosures. This experiment was conducted for 3 days, which minimizes growth and the potential for morphological induction.

At the end of the experiment, all surviving tadpoles and salamanders were returned to the laboratory for measurement of body length and body width (tadpoles) and body length and gape and head width (salamanders). All measurements were made with digital calipers and the total number of surviving larvae was recorded.

\section{Statistical analyses: field experiment}

The proportion of salamanders and tadpoles surviving to the end of the experiment was analyzed with a one-way ANOVA that considered tadpole phenotype (defended, undefended) as a fixed effect. To analyze amphibian morphology at the end of the experiment, we performed MANOVAs on the enclosure means of final body length and body width of tadpoles and final body length and gape and head width of salamanders. When necessary (e.g., a significant trait $\times$ treatment interaction), MANOVAs were followed up with one-way ANOVAs on each trait for each amphibian.

\section{Laboratory experiment}

To obtain a better understanding of the mechanisms underlying the patterns observed in our field experiment, we conducted a laboratory experiment using animals collected from the same ponds. We randomly applied two treatments ("defended" and "undefended") to experimental units (polypropylene tanks, $24.5 \times 37 \times$ $13 \mathrm{~cm}, \mathrm{~L} \times \mathrm{W} \times \mathrm{D})$ filled with $10 \mathrm{~L}$ of aged tap water. Because we were not able to collect as many undefended tadpoles, this treatment was replicated 15 times, whereas the defended treatment was replicated 23 times. We placed two salamanders and 10 undefended tadpoles into each undefended replicate, and two salamanders and 10 defended tadpoles into each defended replicate. The number of amphibians placed in each tank was thus scaled down appropriately to match the densities of animals used in our field experiment. We were also careful to use defended (body length, $11.42 \pm 0.30 \mathrm{~mm}$; body width, $9.07 \pm 0.69 \mathrm{~mm}$; mean $\pm \mathrm{SE}$ ) and undefended (body length, $11.43 \pm 0.28 \mathrm{~mm}$; body width, $6.21 \pm 0.60 \mathrm{~mm}$; mean $\pm \mathrm{SE}$ ) tadpoles that were of similar size to those used in the field experiment. Salamanders were also categorized based on body size (large vs. small) and photographed ventrally and laterally in a glass chamber before placing one individual of each size in each replicate. Resulting images were projected onto a computer monitor to measure salamander body length and gape and head width.

At 24 hours after the start of the experiment, we recorded tadpole activity (moving or stationary) to determine how morphological status (defended or 

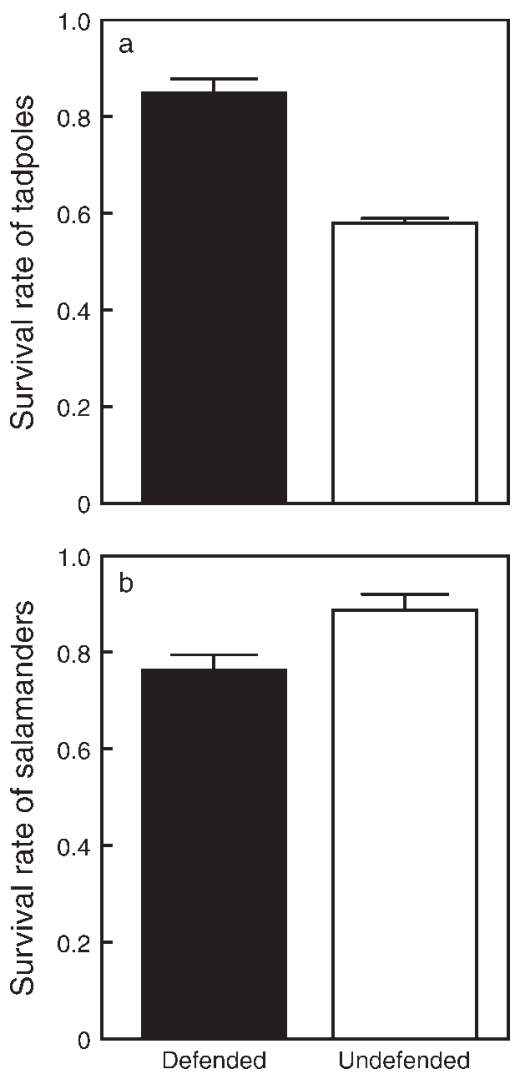

FIG. 3. Survival rate (mean $+\mathrm{SE}$ ) of (a) tadpoles and (b) salamanders under defended and undefended treatments.

undefended) influenced their behavior. After three days, the experiment was terminated and we counted the number of surviving tadpoles and salamanders. We also took ventral and lateral photographs of all surviving salamanders to allow measurement of final morphological traits. In addition, comparison of these photos with our original photos allowed us to identify those individuals (large and/or small) that had survived to the end of the experiment. Individual identification was based on shape and small scars in body and tail parts evident in initial and final photos.

\section{Statistical analyses: laboratory experiment}

We used one-way ANOVAs to examine how tadpole phenotype (defended, undefended) influenced tadpole activity levels after 24 hours and tadpole survivorship at the end of the 3-day experiment. Multiple logistic regression on binomial survivorship data (surviving, cannibalized) examined whether salamander cannibalism was more frequent in the defended vs. undefended treatment. In addition, this analysis examined the importance of (1) the ratio of the initial body length of large vs. small salamanders and (2) the ratio of initial gape width of large salamanders to the initial head width of small salamanders to the likelihood of cannibalism. In this analysis, survivorship was the response variable and tadpole phenotype (defended, undefended) and each trait ratio served as the predictor variables. We found that the effect of the ratio of initial gape width to initial head width on the probability of cannibalism was quite strong, but it is also possible that the higher activity levels of defended tadpoles may have indirectly affected the intensity of cannibalism by influencing salamander activity levels. Hence, we conducted a multiple logistic regression on the binomial survivorship data of salamanders in the defended treatment to determine if tadpole activity levels, in addition to salamander morphology, were important to the probability of cannibalism. Finally, because we found that the gape to head-width ratio was important, we examined whether these variables were influenced by differential gape and head-width growth among our experimental treatments.

\section{Results}

\section{Field experiment}

Tadpole survivorship was significantly $\left(F_{1,6}=85.71, P\right.$ $<0.0001$; Fig. 3a) higher in the defended treatment $(85 \%)$ than in the undefended treatment $(58 \%)$. In contrast, salamander survivorship was significantly $\left(F_{1,6}\right.$ $=8.73, P=0.026)$ lower in the defended treatment $(77 \%)$ than in the undefended treatment (89\%; Fig. 3b).

At the end of the experiment, tadpole morphology continued to differ among the defended and undefended treatments (MANOVA, $F_{1,6}=107.29, P<0.0001$; Fig. 2a), but each trait responded differently (MANOVA, trait $\times$ treatment interaction, $F_{1,6}=330.51, P<0.0001$ ). Tadpoles in the defended treatment still had wider bodies than those in the undefended treatment $\left(F_{1,6}=\right.$ 406.54, $P<0.0001$ ), but tadpoles in the undefended treatment were longer than those in the defended treatment $\left(F_{1,6}=25.00, P=0.0025\right)$.

Salamanders also responded strongly to our experimental treatments with those in the defended treatment having longer bodies, wider heads, and wider gapes than those in the undefended treatment (MANOVA, $F_{1,6}=$ $16.05, P=0.0071$; Fig. 2b). The absence of a significant trait $\times$ treatment interaction $\left(F_{2,5}=0.88, P=0.47\right)$ revealed that all three traits responded similarly to the presence of defended tadpoles.

\section{Laboratory experiment}

Tadpole survivorship was significantly (ANOVA, $\left.F_{1,36}=21.88, P<0.0001\right)$ higher in the defended treatment $(88.3 \%)$ than in the undefended treatment (70.1\%; Fig. 4a) and defended tadpoles were more active (ANOVA, $F_{1,36}=4.59, P=0.039$ ) than undefended tadpoles (Fig. 4b). Multiple logistic regression examined the importance of the ratio of initial body length of the large vs. small salamander and the ratio of the initial gape width of the large salamander to the initial head width of the small salamander. This full model revealed no significant effect of length ratio or any interaction involving length ratio (likelihood ratio tests, all $\chi_{1}^{2} \leq$ 

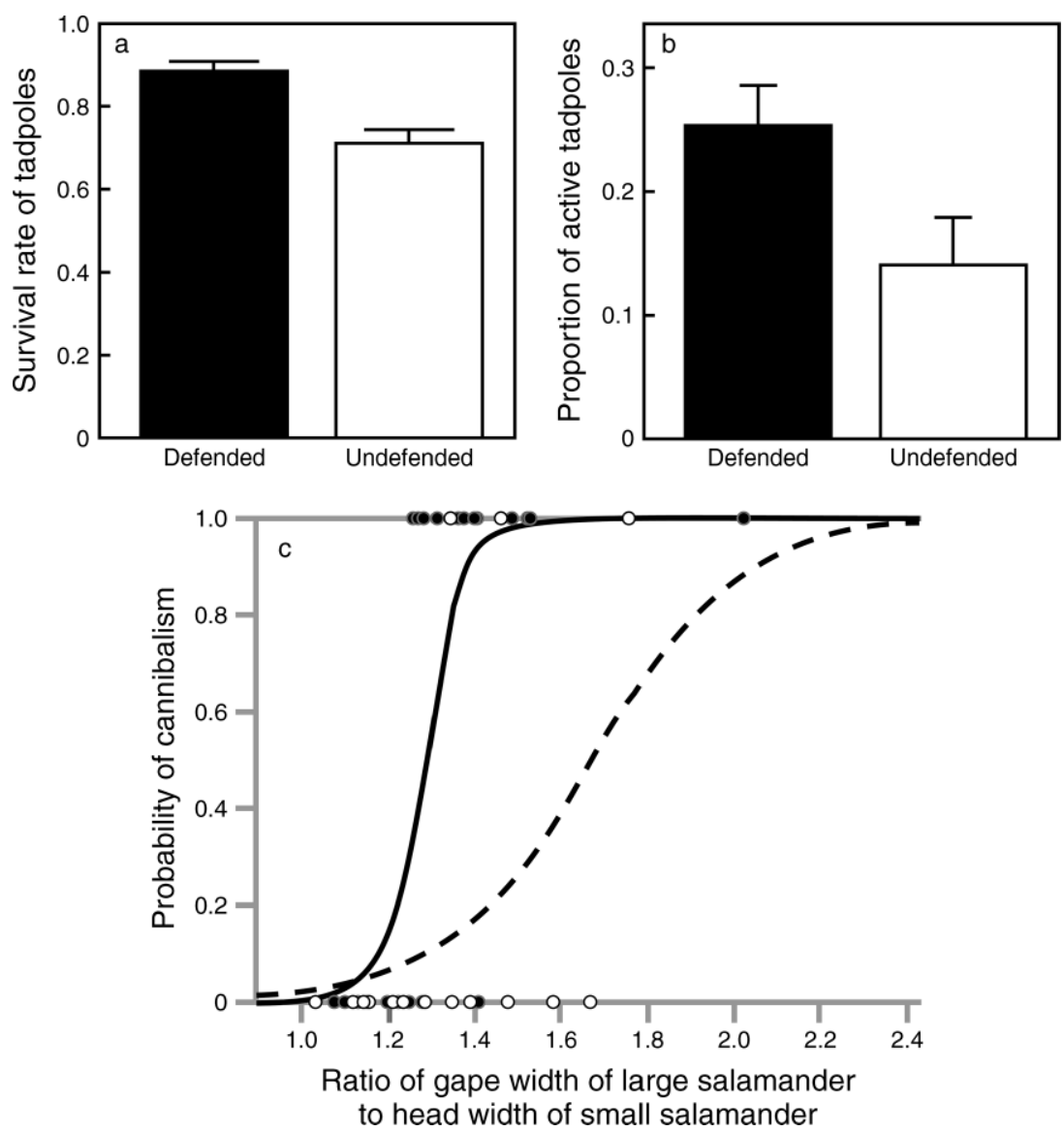

FIG. 4. (a) Survival rate (mean $+\mathrm{SE}$ ) of tadpoles in the defended and undefended treatments, (b) proportion of tadpoles active $($ mean $+\mathrm{SE})$ in the defended and undefended treatments after 24 hours, and (c) the probability of cannibalism occurring in salamanders in the defended and undefended treatments. The $x$-axis represents the ratio of the initial gape width of the large salamander to the head width of the small salamander. The $y$-axis represents the probability of cannibalism by the large salamander on the small salamander. Solid circles and the solid logistic regression line $\left(y=\exp [-26.16+20.40 x] /(1+\exp [-26.16+20.40 x]), R^{2}\right.$ $=0.46)$ represent the defended treatment, whereas open circles and the dashed regression line $(y=\exp [-9.19+5.52 x] /(1+\exp [-9.19$ $+5.52 x]), R^{2}=0.18$ ) represent the undefended treatment.

0.27, all $P \geq 0.51$ ). Hence, we sequentially removed terms involving length ratio from the model ( $P$ values of removed terms were always $P \geq 0.58$ ). This approach yields a final model comprised of tadpole phenotype, the gape-width to head-width ratio, and their interaction. A nonsignificant lack-of-fit statistic $\left(\chi_{30}^{2}=28.45, P=\right.$ 0.5462 ) indicated that the final model would not benefit from additional terms. We found that salamander survivorship was significantly (likelihood ratio test, $\chi_{1}^{2}$ $=9.71, P=0.0018$ ) lower in the defended treatment than in the undefended treatment (Fig. 4c). In 13 out of 23 replicates of the defended treatment, and three out 15 replicates of the undefended treatment, the small salamander was cannibalized. Furthermore, as differences in the gape-width to head-width ratio increased, the probability of cannibalism increased $\left(\chi_{1}^{2}=15.35, P=\right.$ $0.0001)$ and there was a strong trend $\left(\chi_{1}^{2}=3.32, P=\right.$ 0.0685 ) for this effect to be significantly stronger in the defended vs. the undefended treatment (Fig. 4c).
Our analysis exploring the importance of salamander gape-width to head-width ratio and defended tadpole activity levels to salamander survivorship confirmed that the probability of cannibalism increased as differences in the gape-width to head-width ratio increased (likelihood ratio test, $\left.\chi_{1}^{2}=9.95, P=0.0016\right)$. However, we were unable to detect any significant effect of defended tadpole activity level $\left(\chi_{1}^{2}=0.43, P=0.5114\right)$ or its interaction with gape-width to head-width ratio $\left(\chi_{1}^{2}=\right.$ $0.003, P=0.9575)$.

We detected no significant differences in the gapewidth and head-width growth of surviving salamanders among our experimental treatments (MANOVA, $F_{1,58}=$ $1.63, P=0.2062$ ). Hence, interactions between large and small salamanders were not driven by differential growth during the experiment.

\section{Discussion}

Our field experiment found that tadpole survivorship was higher in the defended than in the undefended 
treatment (Fig. 3a). The body length and width of defended tadpoles did not change during the experiment, suggesting that their improved survivorship was directly due to their defended status (bulgy body) at the beginning of the experiment. In contrast, the mortality of undefended tadpoles was $27 \%$ higher, indicating that salamander foraging success is strongly influenced by tadpole morphology. These results agree with those of our laboratory experiment where tadpole mortality was $18.2 \%$ higher in the undefended than in the defended treatments (Fig. 4a).

By the end of the field experiment, undefended tadpoles still had narrower bodies than defended tadpoles (Fig. 2a) and were thus more vulnerable to salamander predation. The lack of change in the body width of undefended tadpoles also indicates that our experimental duration was insufficient for morphological induction to occur. Indeed, the time required for expression of the bulgy morph under similar experimental conditions is $\sim 1$ week (Kishida et al. 2006, 2007). Moreover, at the end of the experiment, surviving tadpoles from the undefended treatment did not have the thickened epithelial tissue that is a diagnostic feature of the defended morphology (Kishida and Nishimura 2004). In contrast, surviving tadpoles in the undefended treatment were significantly longer than those in the defended treatment, suggesting that shorter individuals are also more vulnerable to salamander predation.

Although the increased survival of defended vs. undefended tadpoles in the field likely reflects differences in their respective morphologies, other factors, such as behavior, also may have been operating. We attempted to homogenize potential behavioral effects by conditioning undefended tadpoles to predation risk before initiating our experiment because tadpole experience or naivete can obviously influence survivorship under predation risk. Previous research has shown that other frog tadpole species reduce activity levels to reduce their likelihood of being detected by predators (Skelly 1994, Relyea 2001, Van Buskirk 2002). In our field experiment, the defended tadpoles had experienced salamander predation risk in the pond before we collected them so one might expect them to be less active than naive, undefended tadpoles. Reduced activity levels are expected to reduce both detection by and encounters with salamanders, thereby promoting increased tadpole survivorship. Our laboratory results do not support this hypothesis because defended tadpoles were actually more active than undefended tadpoles (Fig. 4b). Hence, the protection conferred by the bulgy morph may allow tadpoles to forage more actively in the presence of predation risk, whereas undefended tadpoles must compensate for their lack of this defense with lower activity levels that likely reduce their conspicuousness to predators. Such trait compensation, especially between morphological and behavioral traits, is a strategy that can provide fitness advantages in environments where predation risk varies temporally and spatially (DeWitt et al. 1999, Rundle and Brönmark 2001).

Salamander survivorship in the field was reduced by $12 \%$ when defended vs. undefended tadpoles were available as prey (Fig. 3b). This reduction in survivorship likely occurred because the bulgy bodies of defended tadpoles, which are wider than those of salamander conspecifics (Fig. 1b) and thus more difficult to swallow, intensified cannibalism among salamanders. This interpretation is supported by our laboratory results showing that the likelihood of cannibalism increased considerably as the ratio of gape width to head width of large vs. small salamanders increased (Fig. 4c). Although this pattern was evident in both the defended and undefended tadpole treatments, our data suggest that the influence of changes in this ratio on cannibalism was more important in the defended treatment. For example, an $80 \%$ probability of cannibalism occurred at a gape-width to head-width ratio of $\sim 1.3$ in the defended treatment, whereas a gape-width to head-width ratio of $\sim 2.0$ was required before such high cannibalism rates occurred in the undefended treatment. Although undefended tadpoles are also significantly wider than salamander conspecifics (Fig. 1b), overall cannibalism in this treatment was very low (only three out 15 small salamanders were cannibalized). In fact, two of the three cannibalism events we observed in the undefended treatment did not occur until the asymmetry in the gape width and head width of large and small salamanders, respectively, was quite high (a ratio of $\sim 1.4$ or more). Hence, these salamanders appear capable of discriminating between heterospecific and conspecific prey (see Pfennig and Collins 1993 for an example involving kin recognition by cannibalistic salamanders) and such discrimination may be strongly influenced by this asymmetry.

Our analysis highlights the importance of salamander size asymmetries to cannibalistic interactions in the presence of defended tadpoles, but the higher activity of defended tadpoles may have increased salamander activity that, in turn, increased encounters among salamanders and the likelihood of cannibalism. Although this intuitive mechanism may have been operating, further analysis of our laboratory results provided no evidence for it. Indeed, the probability of cannibalism in the presence of defended tadpoles was again strongly influenced by salamander gape-width to head-width ratio, whereas tadpole activity levels had no significant effect. Hence, while we acknowledge that tadpole activity levels have the potential to influence this predator-prey interaction, their effect was surprisingly weak in our laboratory experiment where we were able to measure both variables carefully.

Recent work demonstrates that trait-mediated indirect interactions are important in cannibalistic systems (Rudolf 2006, 2007a, b, 2008), and our study uniquely shows that inducible defenses in prey may be ultimately responsible for their emergence. The existence of trait- 
mediated indirect effects in three-species food chains is well established (Werner and Peacor 2003, Schmitz et al. 2004), as is their influence on trophic cascades (Schmitz et al. 1997, 2004, Trussell et al. 2002, 2006a) and ecosystem function (Trussell et al. 2006b, 2008, Schmitz et al. 2008). Much of this work has focused on the cascading effects of prey habitat or diet shifts in response to predation risk (Schmitz et al. 1997, 2004, Werner and Peacor 2003, Trussell et al. 2006a), but little effort has explored how such indirect effects are transmitted by predator diet shifts in response to induced morphological defenses in prey (but see Raimondi et al. 2000). Our study demonstrates that the heterogeneity in prey edibility created by tadpole inducible defenses causes strong trait-mediated indirect effects in this amphibian system by increasing the intensity of cannibalistic behavior among salamanders and relaxing predation on tadpole prey. Although it is clear that prey heterogeneity and cannibalism can independently affect trophic cascades (Strong 1992, Persson et al. 2003, Rudolf 2007a, b), we suggest that the synergistic effects of tadpole defenses and cannibalism, both of which are ubiquitous in natural systems, may have particularly strong effects on cascade strength.

It is also likely that emergence of cannibalism as a trait-mediated indirect effect of inducible defense can strongly influence amphibian population dynamics. Considerable theory has explored how inducible defenses may regulate population dynamics. In some cases, inducible defenses create negative feedbacks between predator density and prey mortality that stabilize predator-prey dynamics (Ruxton and Lima 1997, Vos et al. 2004b), whereas others have shown that they can destabilize these dynamics (Luttbeg and Schmitz 2000, Kopp and Gabriel 2006). Our results suggest that tadpole defenses and the emergence of cannibalism may ultimately stabilize tadpole-salamander dynamics, as recent models suggest (Rudolf 2007a, b), by relaxing the intensity of salamander predation on tadpoles and increasing salamander mortality rates. However, longer term experiments are needed to fully understand the population and community consequences of cannibalism in this and other systems.

Our study also suggests that tadpole defenses can strongly influence phenotypic evolution in salamanders by intensifying phenotypic selection on salamanders through their enhancement of cannibalistic behavior. In the field, surviving salamanders that were maintained with defended tadpoles were longer, and had wider gapes and wider heads than those maintained with undefended tadpoles (Fig. 2b), suggesting that selection on salamander morphology was intense. Although previous work has shown that predaceous salamander morphs having a large gape can be induced by high tadpole density (Michimae and Wakahara 2002), such induction, as in tadpole defenses, typically requires more time (7-10 days, Kishida et al. 2009) to develop than was possible in our experiment. Moreover, in our laboratory experiment, where we were able to measure individual salamander growth, we did not detect significant differences in the gape and head-width growth of surviving salamanders among our treatments. Hence, because larval salamanders are gape-limited predators, defended tadpoles may directly and indirectly, via their enhancement of cannibalism, drive selection for larger gape and head width in salamanders.

The prevalence of intraguild predation, including cannibalism (Polis 1981, Persson 1999, Rudolf 2006a, 2007a, b, 2008, Rosenheim 2007), in natural systems may be strongly connected to the expression of inducible defenses in numerous taxa (Tollrian and Harvell 1999). We found that cannibalism was intensified by the expression of inducible changes in prey morphology, but predator-induced changes in prey behavior also may cause predators to shift to less vigilant prey, including conspecifics. Regardless of the nature of the defense, we suggest trait-mediated indirect effects, which emerge because of predator diet shifts in response to prey defenses, play a key role in the evolution of predator traits and in maintaining predator diet breadth and cannibalism that, in turn, determine how trophic dynamics unfold in natural systems.

\section{ACKNOWLEDGMENTS}

We are very grateful to Takuya Ikawa, Shinya Kuwano, Yukari Asano, Kaita Nozaki, Saeko Nakagawara, and Akihiko Mougi for their outstanding assistance during the experiment. We also thank two anonymous reviewers for constructive comments that improved this paper. This work was supported by a Grant-in-Aid for Scientific Research (no. 19370005) to K. Nishimura from the Ministry of Education, Culture, Sports, Science, and Technology, and in part by a Grant-in-Aid for a Research Fellow of the Japan Society for the Promotion of Science for young scientists (no. 820076200003) to O. Kishida and grants from the U.S. National Science Foundation (OCE0648525, OCE-0727628) to G. C. Trussell.

\section{Literature Cited}

Agrawal, A. A. 2001. Phenotypic plasticity in the interactions and evolution of species. Science 294:321-326.

Bolker, B., M. Holyoak, V. Krivan, L. Rowe, and O. J. Schmitz. 2003. Connecting theoretical and empirical studies of trait-mediated interactions. Ecology 84:1101-1114.

DeWitt, T. J., A. Sih, and J. Hucko. 1999. Trait compensation and cospecialization: size, shape, and antipredator behaviour. Animal Behavior 58:397-407.

Estes, J. A., and J. F. Palmisano. 1974. Sea otters: their role in structuring nearshore communities. Science 185:1058-1060.

Hairston, N. G., F. E. Smith, and L. B. Slobodkin. 1960. Community structure, population control, and competition. American Naturalist 94:421-424.

Kishida, O., Y. Mizuta, and K. Nishimura. 2006. Reciprocal phenotypic plasticity in a predator-prey interaction between larval amphibians. Ecology 87:1599-1604.

Kishida, O., and K. Nishimura. 2004. Bulgy tadpoles: inducible defense morph. Oecologia 140:414-421.

Kishida, O., and K. Nishimura. 2005. Multiple inducible defenses against multiple predators in anuran tadpoles (Rana pirica). Evolutionary Ecology Research 7:619-631.

Kishida, O., and K. Nishimura. 2006. Flexible architecture of inducible morphological plasticity. Journal of Animal Ecology 75:705-712. 
Kishida, O., G. C. Trussell, and K. Nishimura. 2007. Geographic variation in a predator-induced defense and its genetic basis. Ecology 88:1948-1954.

Kishida, O., G. C. Trussell, and K. Nishimura. 2009. Top-down effects on antagonistic inducible defense and offense. Ecology 90:1217-1226.

Kondoh, M. 2007. Anti-predator defence and the complexitystability relationship of food webs. Proceedings of the Royal Society B 274:1617-1624.

Kopp, M., and W. Gabriel. 2006. The dynamic effects of an inducible defense in the Nicholson-Bailey model. Theoretical Population Biology 70:43-55.

Leibold, M. A. 1989. Resource edibility and the effects of predators and productivity on the outcome of trophic interactions. American Naturalist 134:922-949.

Luttbeg, B., and O. J. Schmitz. 2000. Predator and prey models with flexible individual behavior and imperfect information. American Naturalist 155:669-683.

Matsuda, H., P. A. Abrams, and M. Hori. 1993. The effect of adaptive anti-predator behavior on exploitative competition and mutualism between predators. Oikos 68:549-559.

Michimae, H., and M. Wakahara. 2002. A tadpole-induced polyphenism in the salamander Hynobius retardatus. Evolution 56:2029-2038.

Oksanen, L., S. D. Fretwell, J. Arruda, and P. Niemela. 1981. Exploitation ecosystems in gradients of primary productivity. American Naturalist 118:240-261.

Persson, L. 1999. Trophic cascades: abiding heterogeneity and the trophic level concept at the end of the road. Oikos 85 : 385-397.

Persson, L., A. M. De Ross, D. Claessen, P. Byström, J. Lövgren, S. Sjögren, R. Svanbäck, E. Wahlström, and E. Westman. 2003. Gigantic cannibals driving a whole-lake trophic cascade. Proceedings of the National Academy of Sciences (USA) 100:4035-4039.

Pfennig, D. W., and J. P. Collins. 1993. Kinship affects morphogenesis in cannibalistic salamanders. Nature 362: 836-838.

Polis, G. A. 1981. The evolution and dynamics of intraspecific predation. Annual Review of Ecology and Systematics 12: 225-251.

Polis, G. A. 1984. Age structure component of niche width and intraspecific resource partitioning: can age groups function as ecological species? American Naturalist 123:541-564.

Polis, G. A., and D. R. Strong. 1996. Food web complexity and community dynamics. American Naturalist 147:813-846.

Raimondi, P. T., S. Forde, L. Delph, and C. M. Lively. 2000. Processes structuring communities: evidence for trait mediated interactions through induced polymorphisms. Oikos 91: 353-361.

Relyea, R. A. 2001. Morphological and behavioral plasticity of larval anurans in response to different predators. Ecology 82: 523-540.

Rosenheim, J. A. 2007. Intraguild predation: new theoretical and empirical perspectives. Ecology 88:2679-2680.

Rosenzweig, M. L. 1971. The paradox of enrichment: destabilization of exploitation ecosystems in ecological time. Science 171:385-387.

Rosenzweig, M. L. 1973. Exploitation in three trophic levels. American Naturalist 107:275-294.

Rudolf, V. H. W. 2006. The influence of size-specific indirect interactions in predator-prey systems. Ecology 87:362-371.
Rudolf, V. H. W. 2007a. The interaction of cannibalism and omnivory: consequences for community dynamics. Ecology 88:2697-2705.

Rudolf, V. H. W. 2007b. Consequences of stage-structured predators: cannibalism, behavioral effects, and trophic cascades. Ecology 88:2991-3003.

Rudolf, V. H. W. 2008. Impact of cannibalism on predatorprey dynamics: size-structured interactions and apparent mutualism. Ecology 89:1650-1660.

Rundle, S. D., and C. Brönmark. 2001. Inter- and intraspecific trait compensation of defense mechanisms in freshwater snails. Proceedings of the Royal Society B 268:1463-1468.

Ruxton, D., and S. L. Lima. 1997. Predator-induced breeding suppression and its consequences for predator-prey population dynamics. Proceedings of the Royal Society B 264:409415 .

Schmitz, O. J., A. P. Beckerman, and K. M. O'Brien. 1997. Behaviorally mediated trophic cascades: effects of predation risk on food web interactions. Ecology 78:1388-1399.

Schmitz, O. J., J. H. Grabowski, B. L. Peckarsky, E. L. Preisser, G. C. Trussell, and J. R. Vonesh. 2008. From individuals to ecosystems: toward an integration of evolutionary and ecosystem ecology. Ecology 89:2436-2455.

Schmitz, O. J., V. Krivan, and O. Ovadia. 2004. Trophic cascades: the primacy of trait-mediated indirect interactions. Ecology Letters 7:153-163.

Silliman, B. R., and M. D. Bertness. 2002. A trophic cascade regulates salt marsh primary production. Proceedings of the National Academy of Sciences (USA) 99:10500-10505.

Skelly, D. K. 1994. Activity level and the susceptibility of anuran larvae to predation. Animal Behavior 47:465-468.

Strong, D. R. 1992. Are trophic cascades all wet? Differentiation and donor-control in speciose ecosystems. Ecology 73: $747-754$.

Tollrian, R., and C. D. Harvell. 1999. The ecology and evolution of inducible defenses. Princeton University Press, Princeton, New Jersey, USA.

Trussell, G. C., P. J. Ewanchuk, and M. D. Bertness. 2002. Field evidence of trait-mediated indirect interactions in a rocky intertidal food web. Ecology Letters 5:241-245.

Trussell, G. C., P. J. Ewanchuk, and C. M. Matassa. $2006 a$. Habitat effects on the relative importance of trait- and density-mediated indirect interactions. Ecology Letters 9: 1245-1252.

Trussell, G. C., P. J. Ewanchuk, and C. M. Matassa. 2006 b. The fear of being eaten reduces energy transfer in a simple food chain. Ecology 87:2979-2984.

Trussell, G. C., P. J. Ewanchuk, and C. M. Matassa. 2008. Resource identity modifies the influence of predation risk on ecosystem function. Ecology 89:2798-2807.

Van Buskirk, J. 2002. A comparative test of the adaptive plasticity hypothesis: relationships between habitat and phenotype in anuran larvae. American Naturalist 160:87102.

Vos, M., B. W. Kooi, D. L. DeAngelis, and W. M. Mooij. 2004a. Inducible defenses and the paradox of enrichment. Oikos 105:471-480.

Vos, M., A. M. Verschoor, B. W. Kooi, F. L. Wäckers, D. L. DeAngelis, and W. M. Mooij. 2004b. Inducible defenses and trophic structure. Ecology 85:2783-2794.

Werner, E. E., and S. D. Peacor. 2003. A review of traitmediated indirect interactions in ecological communities. Ecology 84:1083-1100. 